\title{
Symmetrical drug-related intertriginous and flexural exanthema (Baboon syndrome) associated with infliximab
}

\author{
Isil Bulur ${ }^{1}$, Havva Ozge Keseroglu², Zeynep Nurhan Saracoglu¹, Müzeyyen Gönül²
}

1. Department of Dermatology and Venereology, Osmangazi University, Faculty of Medicine, Eskişehir, Turkey;

2. Department of Dermatology and Venereology, Dışkapı Yıldırım Beyazıt Education and Research Hospital, Ankara, Turkey.

Corresponding author:

Isil Bulur, MD

Osmangazi University

Faculty of Medicine

Department of Dermatology

Eskisehir, Turkey

E-mail: isilbulur@yahoo.com

Key words:

adalimumab, adverse event, allergy, erythema, methotrexate, psoriasis, tumor necrosis factor-alpha

\begin{abstract}
Background: Symmetrical drug related intertriginous and flexural exanthema (SDRIFE or Baboon syndrome) is a symmetrical contact dermatitis on inverse regions of the body. The disorder is easily differentiated from other drug eruptions by its typical appearance and lack of other concurrent findings.

Observation: A 50-year-old male patient presented to our clinic complaining of a rash that had developed two days after the tenth infliximab infusion for psoriasis and reoccurred after consecutive infusions. The physical examination revealed a bilateral intergluteal, inguinal, abdominal, axillary, antecubital and neck region macular erythematous rash. There were no other systemic findings. The laboratory values were within normal range. The patient was diagnosed with symmetrical drug-related intertriginous and flexural exanthema associated with infliximab treatment based on dermatological findings, histopathology and the results of the provocation test. The lesions resolved permanently after the patient was swiched from infliximab to adalimumab.
\end{abstract}

Conclusion: Various cutaneous adverse events of anti-tumor necrosis factor alpha treatment have already been reported. The increased use of these agents can lead to a wider variety of drug-induced skin lesions, such as the reported Baboon syndrome. (J Dermatol Case Rep. 2015; 9(1): 12-14)

\section{Introduction}

Infliximab, a humanized antitumor necrosis factor (TNF)- $\alpha$ monoclonal antibody, has been associated with several cutaneous side effects. ${ }^{1}$ We report a patient with previously unreported symmetrical drug-related intertriginous and flexural exanthema (SDRIFE) with remarkable cutaneous symptoms associated with infliximab treatment for chronic plaque psoriasis.

\section{Case Report}

A 50-year-old male patient who had been receiving infliximab therapy for chronic plaque psoriasis presented with a pruritic symmetrical macular erythematous rash of the intergluteal, inguinal, abdominal, axillary, antecubital and neck regions (Fig. 1). The eruption had begun two days after the tenth infliximab infusion and then spontaneously disappeared one week later. The eruption reappeared again after successive infusions, and the lesions became more widespread and lasted longer following each infusion. The medical history of the patient included chronic plaque psoriasis and asthma and the concurrent medications included methotrexate and folic acid. The results of laboratory investigations were within normal limits, except for slight elevation in alanine transaminase $(42 \mathrm{U} / \mathrm{l})$ and gamma glutamyl transferase $(73 \mathrm{U} / \mathrm{L})$. The histopathology of the skin biopsy from the lesion revealed subcorneal pustules, vacuolar degeneration in the dermoepidermal junction, perivascular infiltrates with some neutrophils and eosinophils, and slight epidermal spongiosis in the superficial dermis. The diagnosis of SDRIFE was made clinically and histopathologically. Topical steroid treatment was started and the eruption resolved within two weeks. Despite our recommendations, the patient preferred to continue with infliximab therapy because of his recalcitrant psoriasis and the benign course of the SDRIFE. However, the treatment was continued with adalimumab instead of infliximab because of the development of a more severe eruption on all inverse 


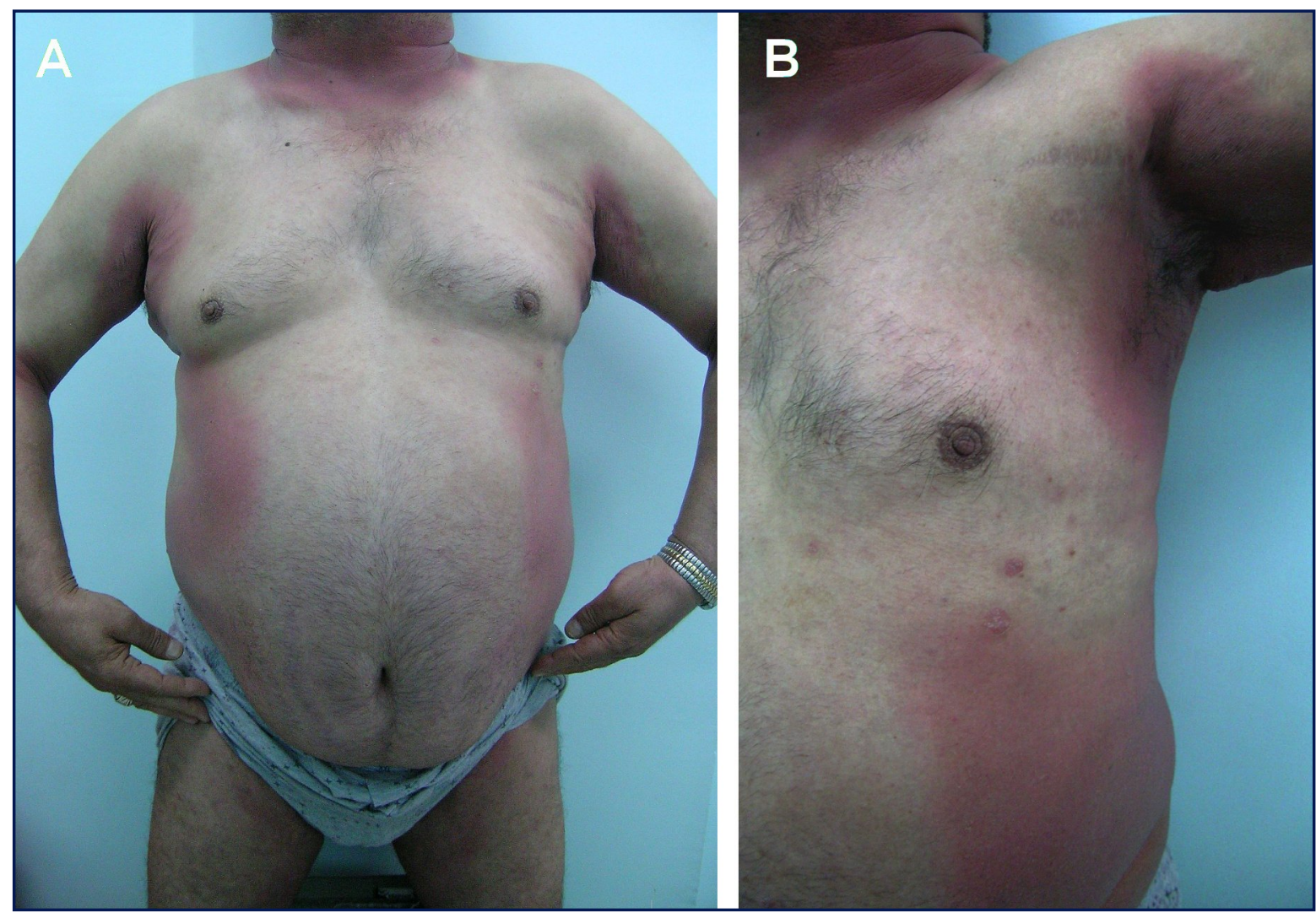

Figure 1

(A) Sharply demarcated erythema on the inguinal, abdominal, axillary, antecubital and neck region;

(B) Close-up view of the region of the $a b$ domen and axillary.

regions including the areas between the fingers and toes that was unresponsive to topical treatment after the sixteenth infusion. We have not observed any skin rash associated with SDRIFE since adalimumab treatment was started.

\section{Discussion}

The term SDRIFE for symmetrical erythema involving the gluteal and intertriginous areas in the absence of systemic involvement and occurring after exposure to systemic drugs was defined by Häusermann et al. ${ }^{2}$ The diagnosis of SDRIFE encompasses five criteria: (1) exposure to a systemically administered drug either for the first time or with repeated doses (excluding contact allergens); (2) sharply demarcated erythema of the gluteal/perianal area and/or V-shaped erythema of the inguinal/perigenital area; (3) involvement of at least another intertriginous/flexural localization; (4) symmetry of the affected areas; and (5) absence of systemic symptoms and signs. ${ }^{2}$ PATCH tests, lymphocyte transformation test (LTT) and drug provocation tests (DPTs) can be used for diagnosis but DPTs remain the gold standard for the determining the offending drug. ${ }^{3}$ Although the histopathology of SDRIFE typically shows a superficial mononuclear perivascular infiltrate with neutrophils and eosinophils, other features, including subcorneal pustules, vacuolar changes and hydropic degeneration in the basal cell layer with subepidermal bullae, and necrotic keratinocytes were also reported. ${ }^{2}$ In our case, the diagnosis of infliximab-associated SDRIFE was based on a typical clinical picture, the histopathology, exclusion of other possible medications, and also a positive provocation test.

The patient was using methotrexate and folic acid concurrently with infliximab therapy, but we did not think of them as the responsible agents as the flares of eruption did not have any temporal relationship with the intake of these drugs. The eruption also disappeared quickly after the cessation of infliximab therapy, although the patient was still on methotrexate and folic acid therapy.

The differential diagnosis of SDRIFE includes fixed drug eruption (FDE), acute generalized exanthematous pustulosis (AGEP) and drug rash with eosinophilia (DRESS). ${ }^{2}$ AGEP and DRESS cause a widespread rash with accompanying systemic changes, while FDE can be easily differentiated from SDRIFE clinically by acral, genital and mucosal roundoval patches or plaques and bullous lesions. ${ }^{2}$ There are a few reports of inverse psoriasis induced by infliximab in the literature and this should also be considered in the differential diagnosis in our patient. ${ }^{4,5}$ On the other hand, the differential diagnosis with psoriasis was made with the clinical findings and the absence of typical psoriasis histopathology.

In our case, it is not possible to known the immunologic mechanism of infliximab associated SDRIFE. SDRIFE is thought to result from a type IV delayed hypersensitivity immune response and acute/delayed hypersensitivity reactions may occur during the treatment period with infliximab. ${ }^{3,6,7}$ Infliximab contains murine sequences at a rate of $25 \%$ and these sequences may be the cause of the immunological reactions. ${ }^{8,9,10}$ We also think that TNF- $\alpha$ inhibitor therapy may lead to aberrant IFN- $\alpha$ expression and SDRIFE lesions could be induced with a mechanism similar to that of pustular dermatitis. ${ }^{5,11}$ 


\section{Conclusions}

In conclusion, several cutaneous adverse reactions have been reported previously in patients receiving treatment with infliximab. ${ }^{1}$ However, this is the first reported case of SDRIFE associated with infliximab to the best of our knowledge.

\section{References}

1. Mocci G, Marzo M, Papa A, Armuzzi A, Guidi L. Dermatological adverse reactions during anti-TNF treatments: focus on inflammatory bowel disease. J Crohns Colitis. 2013; 7: 769-779. PMID: 23453887.

2. Häusermann P, Harr T, Birchner AJ. Baboon syndrome resulting from systemic drugs: is there strife between SDRIFE and allergic contact dermatitis syndrome? Contact Derm. 2004; 51: 297-310. PMID: 15606657.

3. Tan SC, Tan JW. Symmetrical drug-related intertriginous and flexural exanthema. Curr Opin Allergy Clin Immunol. 2011; 11: 313-318. PMID: 21659857.

4. Nuño-González A, Dehesa L, Ricotti C, Kerdel F. Flexural or inverse psoriasis in a patient with hidradenitis suppurativa receiving treatment with infliximab. Actas Dermosifiliogr. 2012; 103: 936-937. PMID: 23149048.
5. Nguyen K, Vleugels RA, Velez NF, Merola JF, Qureshi AA. Psoriasiform reactions to anti-tumor necrosis factor $\alpha$ therapy. J Clin Rheumatol. 2013; 19: 377-381. PMID: 24048106.

6. Cheifetz A, Mayer L. Monoclonal antibodies, immunogenicity, and associated infusion reactions. Mt Sinai J Med. 2005; 72: 250-256. PMID: 16021319.

7. Kleyn CE, Griffiths CE. Infliximab for the treatment of psoriasis. Expert Opin Biol Ther. 2006; 6: 797-805. PMID: 16856801.

8. Steenholdt C, Svenson M, Bendtzen K, Thomsen OØ, Brynskov J, Ainsworth MA. Severe infusion reactions to infliximab: aetiology, immunogenicity and risk factors in patients with inflammatory bowel disease. Aliment Pharmacol Ther. 2011; 34: 5158. PMID: 21535447.

9. Miehsler W, Novacek G, Wenzl H, Vogelsang H, Knoflach $P$, Kaser A, Dejaco C, Petritsch W, Kapitan M, Maier H, Graninger W, Tilg $\mathrm{H}$, Reinisch W; Austrian Society of Gastroenterology and Hepatology. A decade of infliximab: the Austrian evidence based consensus on the safe use of infliximab in inflammatory bowel disease. J Crohns Colitis. 2010; 4: 221-256. PMID: 21122513.

10. Lecluse LL, Piskin G, Mekkes JR, Bos JD, de Rie MA. Review and expert opinion on prevention and treatment of infliximabrelated infusion reactions. Br J Dermatol. 2008; 159: 527-536. PMID: 18627374.

11. Kavala M, Zindancı I, Türkoglu Z, Can B, Kocatürk E, Senol S, Topaloglu F. Acute generalized exanthematous pustulosis induced by etanercept: another dermatologic adverse effect. Case Rep Dermatol Med. 2013; 2013: 601412. PMID: 23573431. 